\title{
Liquid Chromatography-Tandem Mass Spectrometry Method for Determination of Paclitaxel in Human Plasma
}

\author{
G. Rajender* and N.G.B. Narayana
}

Research \& Development Division, Relisys Medical Devices Ltd, Hyderabad, INDIA

\begin{abstract}
A rapid and sensitive liquid chromatography - tandem mass spectrometry (LC-MS/MS) method for determination of the Paclitaxel in human plasma was developed and validated.Placitaxel was extracted from plasma by a two-step extraction procedure with using of chloroform as Liquid -Liquid extractive organic solvent. LC-MS/MS analysis using electro-spray ionization (ESI+) was performed on Phenomenex C-18 Column $(250 \times 1.5 \mu)$ using as acetonitrile, water $(80: 20+0.1 \%$ acetic acid) as mobile phase. The method has a flow rate of $0.8 \mathrm{ml} / \mathrm{min}$. Retention of Paclitaxel was 4.60 minutes.An excellent linearity $\left(r^{2}\right.$.099) between the peak ratios and Paclitaxel concentrations over the range of 10-100 $\mathrm{ng} / \mathrm{ml}$ of plasma was studied. The lower limit of detection for Paclitaxel on mass was $10 \mathrm{pg} / \mathrm{mL}$. There was about 100 percent of Paclitaxel was recovered in extracted samples. The study of Paclitaxel standard and extraction standard calibration curves were useful in pharmacokinetics analysis.
\end{abstract}

Keywords: Liquid chromatography-tandem mass spectrometry; Paclitaxel; Plasma: Chloroform extraction

\section{Introduction}

Paclitaxel (Figure 1) is a natural product extracted from western yew tree, and takes its name from the Latin, Taxus brevifolia .It blocks mitosis by stabilizing the microtubules in cancer cells. During normal cell division, the microtubules are polymerized at the beginning of mitosis to be able to separate the daughter chromosomes.Then they depolymerise back to tublin. Paclitaxel stops this depolymerisation so that the cells become filled with microtubules and cannot divide again. Paclitaxel is active against a number of cancers e.g. of the ovaries, breast, lung and stomach (1).

Paclitaxel is virtually insoluble in water and in most pharmaceutically acceptable solvents; such that it has poor oral bio availability therefore it is mainly administered by the intravenous (IV) route. Currently the vehicle to administer Paclitaxel by IV is a mixture of Ctenophore EL (polyethoxylated castor oil) and ethanol. This vehicle provokes adverse effects, such as hypersensitivity [2].

Various experiments have been performed to improve the bioavailability of this drug such as binding with polymers [3], coadministration with Cyclosporine A [4] or administration by liposome's [5]. To be able to compare the effectiveness of different formulations, Paclitaxel concentrations need to be measured with a fully validated method with sufficient sensitivity to measure plasma levels from the "poor" formulation as well as the improved formulation Paclitaxel is rarely used as monotherapy but is administered with other anticancer drugs to create a synergy of action allowing Paclitaxel doses to be

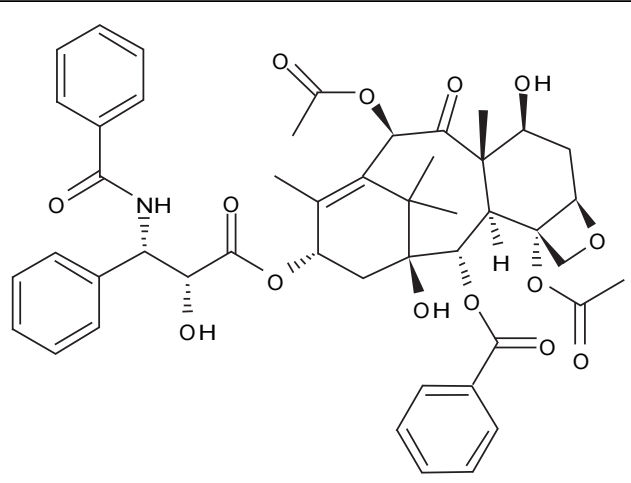

Figure 1: Chemical strucature of Paclitaxel. lowered, consequently the side effects decrease. For example, Paclitaxel has demonstrated synergy with the pyrimidine analogue5flurouracil (5FU) [6], gemcitabine [7], some anticonvulsants [8] and flavopirido [9]. Again, when Paclitaxel is given with other drugs, there is a need to evaluate pharmacokinetic parameters using a validated assay, with high specificity in order that co-administered drugs and/or metabolites do not interfere with the measurement of Paclitaxel. Various HPLC methods for validation of Paclitaxel was studied [14]. A review of early methods of determination of paclitaxol concentrations has been presented by Sparreboom et al. [2]. The aim of the present study was investigate the Paclitaxel eatracted samples, and standard samples with high sensitivity,accuracy was studied wit Liduid Chromatography- tandaem massspectrometry method which is use full for Pharmacokinetic analysis.

\section{Material and Methods}

\section{Chemicals and reagents}

Paclitaxel was provided by Dr.Reddys Research laboratory, hyderabad-INDIA).HPLC grade acetonitrile \& chloroform were procured from Merck. Ultra pure water was obtained from Mille - Q water (S G ultra pure waters system).

\section{Solutions}

Stock solution of Paclitaxel was prepared by dissolving $1 \mathrm{mg}$ of Paclitaxel in $1 \mathrm{ml}$ of acetonitrile. Standard solutions were obtained by diluting this solution with acetonitrile to give the final concentrations over the range of $10-100 \mathrm{ng} / \mathrm{ml}$ for preparation of the standard calibration curve. Acetonitrile, water $(80: 20+0.1 \%$ acetic acid) was prepared for mobile phase and as well for reconstitution of Paclitaxel extracted sample from plasma.

*Corresponding author: G. Rajender, Research \& Development Division, Relisys Medical Devices Ltd, Hyderabad, INDIA, E-mail: dr.grajender@gmail.com

Received August 20, 2010; Accepted September 21, 2010 Published Septembe 21,2010

Citation: Rajender G, Narayana NGB (2010) Liquid Chromatography-Tandem Mass Spectrometry Method for Determination of Paclitaxel in Human Plasma. Pharm Anal Acta 1:101. doi:10.4172/2153-2435.1000101

Copyright: (c) 2010 Rajender G, et al. This is an open-access article distributed under the terms of the Creative Commons Attribution License, which permits unrestricted use, distribution, and reproduction in any medium, provided the original author and source are credited. 


\section{Chromatographic condition}

LC-MS/MS, Quattro micro API, triple quadrupole. Mass Lynx software, version 4.1. Consisted series of 2695 separation module and PDA (2996) detector all from Waters (Milford, MA, USA). Separation was achieved using phenomenax C-18 column (250x4.60 mm-5microns). The mobile phase contains $0.1 \%$ Acetic acid $(80: 20+0.1 \%$ Acetic acid) was prepared and degassed. Chromatographic separations were performed at $30^{\circ} \mathrm{C}$. The flow rate was set to $0.8 \mathrm{ml} / \mathrm{min}$.

\section{LC-MS parameters}

Source (Es +): Capillary (KV): 4.0; Cone (V): 40; Extractor (V): 1.0; RF lens (V): 0.1 ; Source Temperature $\left({ }^{\circ} \mathrm{C}\right)$ : 120 ; Desolvation Temperature $\left({ }^{\circ} \mathrm{C}\right)$ : 450 ; Cone gas flow $(\mathrm{L} / \mathrm{Hr}): 50$; Desolvation gas flow (L/Hr): 650

Analyzer: $\mathrm{LM}_{1}$ Resolution: $12.0 ; \mathrm{HM}_{1}$ Resolution: 12.0; Ion energy ${ }_{1}$ : 1.0; Entrance: 2.0; Collision: 30.0; Exit: 2.0; $\mathrm{LM}_{2}$ Resolution: 12.0; $\mathrm{HM}_{2}$ Resolutions: 12.0 ; Ion energy $y_{2}: 1.0$; Multiplier (V): 650 ; Syringe pump flow (lit/min): 10; Gas cell pirani pressure (mbar): ^ 1e-4 Bars

\section{Sample preparation}

Plasma sample were obtained from healthy volunteers. For this samples $1 \mathrm{~mL}$ of Plasma was added into test tube and $50 \mu \mathrm{l}$ of standard (10, 20, 40, 60,80and 100ng) solution of Paclitaxel was added. These tubes are vortex for $1 \mathrm{mint}$.After adding $1 \mathrm{ml}$ of chloroform to the test tubes, vortexed for 2 mints and centrifuged for $5 \mathrm{~min}$ at $20^{\circ} \mathrm{C}$ and $1500 \mathrm{~g}$. That chloroform layer was completely removed and transferred to a clean test tube and evaporated to dryness at $37^{\circ} \mathrm{C}$. These samples were reconstituted with mobile phase $80: 20+0.1 \%$ Acetic acid.

\section{Sample extraction}

Samples were extracted with liquid-liquid (L-L)method . Two of the common organic solvents used for the L-L extraction are methyl acetate [10] and methyl tertiary butyl ether (MTBE) [5]. The few articles mentioning SPE demonstrate the use of non-polar sorbents (C18 cartridges [11) or polar sorbents (Cn cartridges, [12, 13]. Here we used chloroform as extraction organic solvent. Once the drug has been extracted from the plasma to chloroform, then the drug sample was evaporated at $37^{\circ} \mathrm{C}$. LC-MS/MS system and separation is performed generally by reversed phase HPLC. $50 \mu \mathrm{l}$ of each sample

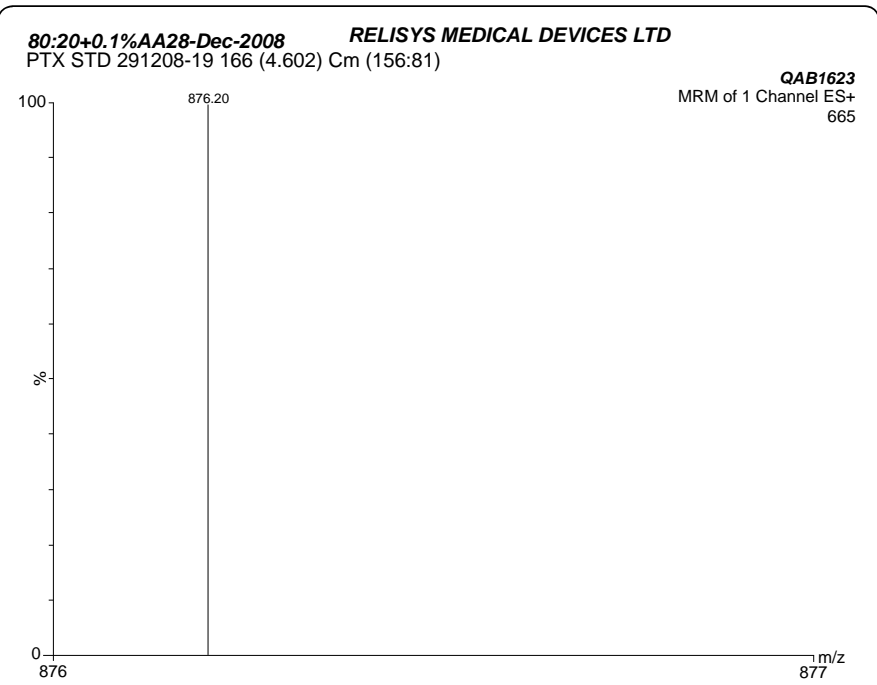

Figure 2: $\mathrm{ESI}^{+}$mode, Paclitaxel protonated molecules $[\mathrm{M}+\mathrm{H}]^{+}$of $\mathrm{m} / \mathrm{z} 876.20$.
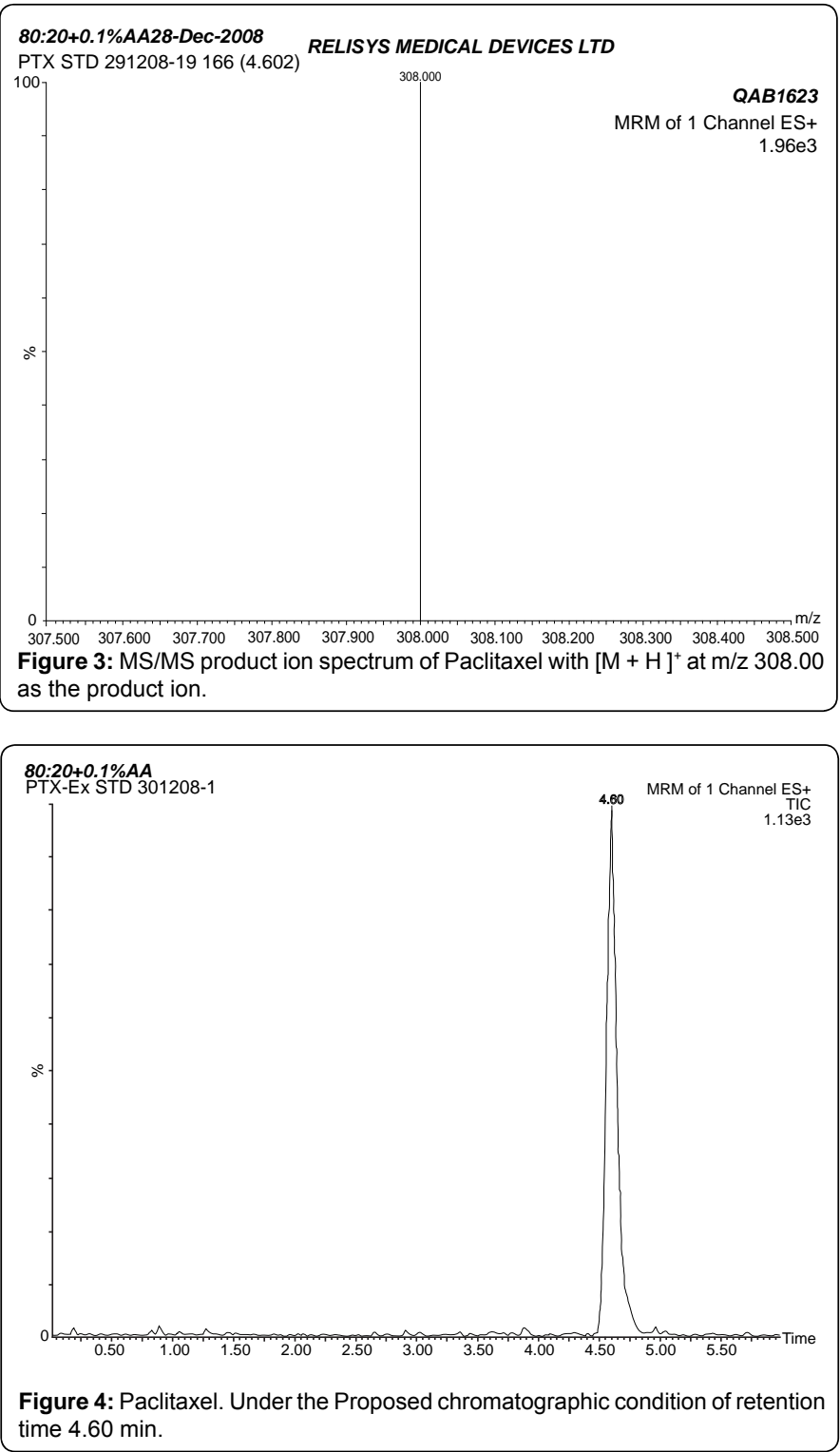

was injected to LC-MS/MS. The experiment was repeated on three consecutive days

\section{Quantification}

Calibration standards of Paclitaxel were prepared by spiking $1 \mu \mathrm{g}$ Paclitaxel standard to $1 \mathrm{ml}$ of blank human plasma to give final concentrations over the range of $1-100 \mathrm{ng} / \mathrm{mL}$. Calibration curves were prepared by plotting the measured peak area ratios of Paclitaxel vs. concentration of standard samples. The intra day (with in run) and interday (between run) accuracy and precision of the method was determined by measuring sample of Paclitaxel standards solution (10, $20,40,60,80,100 \mathrm{ng} / \mathrm{ml}$ ) on three separate days.

\section{Results}

In the present study simple liquid-liquid extraction procedure was performed. The extraction efficiency was increased when liquidliquid extraction (LLE) using dichloro-methane, Petroleum ether, methanol, chloroform extraction procedure was used. The extraction recovery was increased when chloroform as extractive solvent. It was found that all solvents gave high extraction efficiency for Paclitaxel. 
Citation: Rajender G, Narayana NGB (2010) Liquid Chromatography-Tandem Mass Spectrometry Method for Determination of Paclitaxel in Human Plasma. Pharm Anal Acta 1:101. doi:10.4172/2153-2435.1000101

Quantify Compound Summary Report MassLynx 4.1

Dataset: $\quad$ C:MassLynx\PTXSTD151107.PROIPTX-100-10ng-10109.qld

Last Altered: $\quad$ Wednesday, December 31, 2008 11:09:36 India Standard Time

Printed: $\quad$ Thursday, January 08, 2009 12:29:18 India Standard Time

Method: C:|MassLynx|PTXSTD151107.PRO|MethDB|PTX60208-2.mdb 04 May 2008 22:26:13

Calibration: 31 Dec 2008 11:09:36

Compound name: PTX STD

Correlation coefficient: $r=0.995033, r^{\wedge} 2=0.990090$

Calibration curve: $6.4653 * x+6.31373$

Response type: External Std, Area

Curve type: Linear, Origin: Include, Weighting: Null, Axis trans: None

\begin{tabular}{|c|c|c|c|c|c|c|c|c|}
\hline & \# Name & Std.Conc & $\mathrm{RT}$ & Area & IS Area & Response Detecti... & $\mathrm{ng}$ & \%Dev Mod.U... Mod.C... \\
\hline & 1 PTX STD 29120... Standard & 100.000 & 4.63 & 659.553 & & $659.553 \mathrm{bb}$ & 101.0 & 1.0 \\
\hline & 2 PTX STD $29120 \ldots$ Standard & 80.000 & 4.63 & 483.926 & & $483.926 \mathrm{bb}$ & 73.9 & -7.7 \\
\hline & 3 PTX STD 29120... Standard & 60.000 & 4.63 & 429.126 & & $429.128 \mathrm{bb}$ & 65.4 & 9.0 \\
\hline & 4 PTX STD $29120 \ldots$ Standard & 40.000 & 4.63 & 282.728 & & $282.728 \mathrm{bb}$ & 42.8 & 6.9 \\
\hline & 5 PTX STD 29120... Standard & 20.000 & 4.63 & 118.322 & & $118.322 \mathrm{bb}$ & 17.3 & -13.4 \\
\hline & 6 PTX STD 29120... Standard & 10.000 & 4.63 & 74.783 & & $74.783 \mathrm{bb}$ & 10.6 & 5.9 \\
\hline
\end{tabular}

\section{Quantify Calibration Report $\quad$ MassLynx 4.1}

Dataset: C:MassLynxIPTXSTD151107.PRO\PTX-100-10ng-10109.qld

$\begin{array}{ll}\text { Last Altered: } & \text { Wednesday, December 31, 2008 11:09:36 India Standard Time } \\ \text { Printed: } & \text { Thursday, January 08, 2009 12:29:18 India Standard Time }\end{array}$

\section{Method: C:|MassLynx|PTXSTD151107.PRO|MethDB|PTX60208-2.mdb 04 May 2008 22:26:13} Calibration: 31 Dec 2008 11:09:36

\section{Compound name: PTX STD}

Correlation coefficient: $r=0.995033, r^{\wedge} 2=0.990090$

Calibration curve: $6.4653{ }^{*} x+6.31373$

Response type: External Std, Area

Curve type: Linear, Origin: Include, Weighting: Null, Axis trans: None
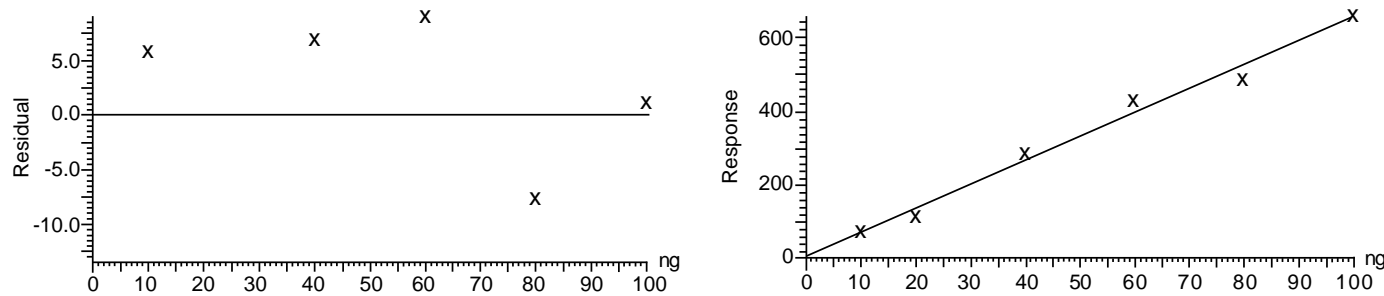

Figure 5: Calibration curves (A) List of samples in nano grams for calibration (B) The linearity of Paclitaxel calibration curve.

In this study, $\mathrm{ESI}^{+}$was chosen as the ionization source. Total Ion Current (TIC)was increased when adding if Acetic acid $0.1 \%$ to the mobile phase. It was found that the signal intensity of Paclitaxel in human plasma was high using $\mathrm{ESI}^{+}$source. The $\mathrm{ESI}^{+}$source provided satisfactory data on method validation and subsequent quantitation for plasma samples from healthy volunteers. $\mathrm{ESI}^{+}$mode, Paclitaxel formed predominantly protonated molecules $[\mathrm{M}+\mathrm{H}]^{+}$of $\mathrm{m} / \mathrm{z} 876.20$ (Figure 2). The paclitaxel parent molecule was fragmented while applying collision energy as organ, and formed product ions (Figure 3). Product ion is necessary for quantification of paclitaxel.

This method showed on interfering peaks of any other contamination other than Paclitaxel. Under the Proposed chromatographic condition of paclitaxel retention time $4.60 \mathrm{~min}$
(Figure 4). The extracted samples chromatograms were co-related to the standard. The recovery of paclitaxel in different concentration, determined by comparing peak areas from extracted standard samples. There was about more than $99 \%$ of Paclitaxel was recovered from plasma.

\section{Calibration}

Standard Calibration curve for Paclitaxel in different range of concentrations $(10,20,40,60,80,100 \mathrm{ng} / \mathrm{ml})$ in plasma were prepared. The linearity of calibration curve $\left(r^{2}>0.99\right)$ (Figure 5) concentration ranges are investigated. The calibration curve obtained in plasma sample Calibration curve: $4.4653 \% \mathrm{X}+6.31373$. The accuracy and precision was determined by preparing with 5 samples of Paclitaxel at concentrations of $1,10,20,40,60,80,100 \mathrm{ng} / \mathrm{ml}$ in plasma on three 
separate days. According to the intraday (within- run) and interday (between- run) a very good data of accuracy and precision were observed over the entire concentration range.

\section{Method validation}

Selectivity: This method was specific for Paclitaxel with no interference of peaks at the retention time of Paclitaxel.

Linearity: A linear range equation was judged to produced the best fit the concentration / response relationship. The regression type was $1 /$ concentration and peak area for a 6-point calibration curves were found to linear from 10 nano grams to 100 nano grams. The goodness to fit for concentrations were consistently greater than 0.99 during the course of validation and study period.

Recovery: The percentage of recovery of Paclitaxel from PBS is observed as more than $99 \%$.

Accuracy: The accuracy of the assay was defined as the absolute value of the ratio of the back calculated mean values of the quality control samples to their normal values, expressed as percentage. Within the batch, accuracy range from $90.54 \%$ to 112.93 . Between the batch , accuracy range from $97.70 \%-105.37 \%$.

Precision: The precision of the assay was measured by the percent coefficient of variation over the concentration range of quality control samples, respectively of Paclitaxel during the course of the study. Within batch Precision ranged from $0.04 \%$ to $15.19 \%$.Between batch Precision ranged from $3.84 \%$ to $10.49 \%$.

Stability: Short-term stability of Paclitaxel was determined by comparing the mean of the area responses obtained from 3 replicate analysis of aqueous standard $(40 \mathrm{ng} / \mathrm{ml})$ at 0.0 hours and after 6.0 hours. Ratio of means of area was $102.5 \%$. Witch is within the acceptance range of $90-110 \%$.

Long- term stability of Paclitaxel was determined by comparing the mean of the area responses obtained from 3replicate analysis of aqueous standard $(80 \mathrm{ng} / \mathrm{ml})$ after 12 days and freshly prepared aqueous standard. Ratio of means of area was $101.7 \%$. Witch is within the acceptance 1 rangeof $90-110 \%$.

\section{Discussion}

In the present study the importance of LC-MS/MS techniques for determination of paclitaxel in biological fluids were observed. Paclitaxel was extracted from aqueous medium into an organic solvent. The LC-MS/MS method was validated and subsequently used to analyze samples from a comparison study.

The LC-MS/MS method demonstrated high specificity because only ions derived from the analytes of interest were monitored. The selectivity towards endogenous plasma matrix was tested in different batches of human plasma by analysis blanks and samples at LLOQ levels. Paclitaxel chromatograms indicate no significant visible interference at the expected retention time of the analyte since Paclitaxel was modified to elute in a region where visible interference is not observed. The retention time of Paclitaxel was $4.6 \mathrm{~min}$. This is the shortest total run time $(6.0 \mathrm{~min})$ for determination of Paclitaxel in plasma.

In the present study, a two step extraction procedure was described using chloroform as extracting solvent. The accuracy and precision for this sample and rapid procedure support the reliability of the assay for the determination of paclitaxel in biological fluids and for pharmaco kinetics studies.

\section{Conclusion}

A two step extraction procedure for paclitaxel from human plasma and an improved method for determination of paclitaxel were reported. The LCMS/MS method need a cleaner extract at it less specific and any endogenous compounds with the same retention time and similar maximum of absorption would interfere with the assay, UV detection can be used and an adequate PK prophile can obtained with a method validated between 10 and 100nano gram/ $\mathrm{mL}$ [13].

In this study, we reported a newly developed LC-MS/MS method for the determination of Paclitaxel in human plasma. The LC-MS/MS technique is suitable for determination of small amount of Paclitaxel with good accuracy and reproducibility. The sample pretreatment was easy and fast using chloroform extraction. $\mathrm{ESI}^{+}$technique with satisfactory mass spectral response generated for paclitaxel parent molecule. This method was successfully applied to determined Paclitaxel concentration in human plasma, for processing of multiple samples in a limited amount of time for pharmacokinetics studies.

\section{References}

1. National cancer institute facts sheets, Oncolink website (http:/www.oncolink com)

2. Sparreboom A, van Tellingen O, Nooijen WJ, Beijnen JH (1998) Preclinical pharmacokinetics of paclitaxel and docetaxel. Anticancer Drugs 9: 1-17.

3. Nannan Panday VR, Meerum Terwot JM, Ten Bokkel Huinink WW (1998) The role of pro drug therapy in the treatment of cancer. Proc Am Soc Clin Oncol 17 $742 a$.

4. Schellens JHM, Meeru JM, Bokke HWW, Rosing H, Tellingen O, et al. (1998) Cyclosporin A (CsA) strongly enhances oral Bioavailability of Paclitaxel (Pac) in cancer patients. Pro Am Soc Clin Oncol 17: 717a.

5. Sharma A, Conway WD, Staudinger RM (1994) Reverse-phase high- performance liquid chromatographic determination of taxol in mouse plasma. J Chromatogr 655: 315-319.

6. Patton JF, Suzanne J, Fanthony G, Haimworth J, Willent N, et al. (2000) Weekly Paclitaxel prolongated oral Eniluracil/ 5-Flurouracil: Results of a phasecombination study. Proc Am Soc Clin Oncol 19: 746a.

7. Rinaldi DA, Lormand N, Brierre J, Cole J, Stagg P, et al. (2000) A Phase I trial of Gemcitabine (GEM) and Paclitaxel (PAc) in patients with advanced solid tumors, Administered Every 21 days(LOA-2). Proc Am Soc Clin Oncol 19: 848a.

8. Kuhn J, Rizzo J, Chang S, Schold C, Spence A, et al. (1997) Effect of anticinvulsants (ACs) on the phromacokinetics(PK) and metabolic profile of Paclitaxel. American society of clinical oncology 16: 224a.

9. Schwartz GK, Kaubish A, Saltz L, Lison D (2000) Phase I trial of sequential Paclitaxel and the cyclin dependent kinase inhibitor flavopiridol. Proc Am Soc Clin Oncol 18: 160a.

10. El-Yazigi A, Yusuf A (1995) High Performance liquid chromatographic procedure for the quantitative determination of paclitaxel (Taxol) in human plasma. Ther Drug Monit 17: 511-515.

11. Masc H, Hiraoka M, Suzuki A (1994) Yakugaku Zasshi. 5: 351-355.

12. Huizing MT, Sparreboom A, Rosing H (1995) Quantification of paclitaxel metabolitesin human plasma. J Chromatogr 674: 261-268.

13. Willey TA, Bekos EJ, Gaver RC, Duncan GF, Tay LK, et al. (1993) A high performance liquid chromatographic procedure for the quantitative determination of paclitaxel in human plasma. J Chromatogr 621: 231-238.

14. Rajender G, Narayanan NGB (2009) Sensitive and Validated HPLC method for determination Paclitaxel in human serum. Indian J SciTechnol 5: 52-54. 